\title{
Wichard von Moellendorff (1881-1937)
}

\author{
Materialprüfer, Metallforscher, Wirtschaftspolitiker
}

\author{
Von Jürgen Evers, Ulrich von Möllendorff und Ulrich Marsch
}

\begin{abstract}
Überblick
Wichard von Moellendorff ist bekannt als „konservativ-sozialistischer“ Wirtschaftspolitiker, der während des Ersten Weltkrieges und in der frühen Weimarer Republik hervortrat. Kaum bekannt sind dagegen seine Leistungen als Ingenieur und Forscher. Er errichtete und leitete bis 1914 das erste Werkstoffprüf- und -forschungslabor der Allgemeinen Elektricitäts-Gesellschaft (AEG) und führte dort grundlegende kristallographisch-technische Untersuchungen an Metallen und Legierungen durch. Als Leiter des Staatlichen Materialprüfungsamtes und zugleich des Kaiser-Wilhelm-Instituts für Metallforschung baute er ab 1923 unter schwierigen Bedingungen eine in der Welt führende Forschungsstätte auf.
\end{abstract}

\begin{abstract}
Wichard von Moellendorff is known as a „conservative socialist“ economist and politician prominent during World War I and the early Weimar Republic. Hardly known, however, are his achievements as an engineer and researcher. He organized and directed until 1914 the first materials testing and research laboratory of Allgemeine Elektricitäts-Gesellschaft (AEG), where he performed fundamental crystallographic and technological investigations on metals and alloys. As director of the governmental materials testing authority and simultaneously of the Kaiser Wilhelm institute of metals research, he established from 1923, in difficult conditions, an internationally leading research institution.
\end{abstract}

$* * *$

\section{Das Metall-Laboratorium der Allgemeinen Elektricitäts-Gesellschaft} Gegen Ende des 19. Jahrhunderts entwickelte die elektrotechnische Industrie wie vor ihr andere Branchen - etwa die Hütten- oder die chemische Industrie - Strategien und Strukturen zur Kontrolle der für sie relevanten Technologien und Materialien und für weitere Erfindungen. Forschungs- und Prüflaboratorien erwiesen sich dabei als äußerst erfolgreiche Neuerungen. ${ }^{1}$ Wäh-

1 U. Marsch, Zwischen Wissenschaft und Wirtschaft: Industrieforschung in Deutschland und Großbritannien 1880 -1936, Paderborn, München, Wien, Zürich 2000; C. Reinhardt, 


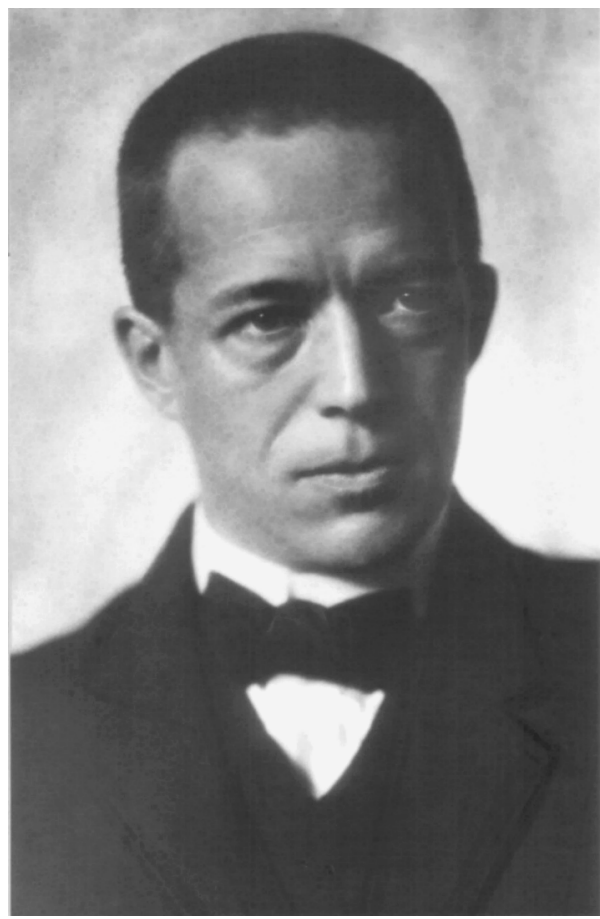

Abb. 1: Wichard von Moellendorff (Quelle: Bundesanstalt für Materialforschung und -prüfung)

rend aber z.B. die chemischen Firmen fast identische Strukturen ihrer Laboratorien aufwiesen, unterschieden sich die beiden deutschen elektrotechnischen Kontrahenten, Siemens und Allgemeine Elektricitäts-Gesellschaft (AEG), darin ganz erheblich voneinander. Entsprechend der Tradition des „Erfinder-Unternehmers“ hatten bei Siemens interne Forschungen und Versuche von Anfang an hohe Priorität. Die AEG hingegen, von Emil Rathenau ursprünglich nicht zur Umsetzung eigener Erfindungen, sondern zur Nutzung der Edisonschen Beleuchtungspatente gegründet, beschritt ähnliche Wege erst viel später und hatte ein grundlegend anderes Verständnis der Bedeutung wissenschaftlicher Forschung. ${ }^{2}$ Um 1900 setzte die AEG dazu an, Siemens an Größe und Bedeutung zu überholen. ${ }^{3}$ Aber erst 1908 entschloss sich ihr Vorstand, im AEG-Kabelwerk Oberspree in Oberschöneweide bei Berlin, dem damals größten Metallverarbeitungswerk Deutschlands, ein „Metall-Laboratorium nach dem Vorbild öffentlicher Prüfämter“ zu grün-

Forschung in der chemischen Industrie: die Entwicklung synthetischer Farbstoffe bei BASF und Hoechst, 1863 bis 1914, Freiberg 1997.

2 R. W. Serchinger, Walter Schottky und die Forschung bei Siemens, in: I. Schneider, H. Trischler u. U. Wengenroth (Hg.), Oszillationen. Naturwissenschaftler und Ingenieure zwischen Forschung und Markt, München 2000, S. 167-210, hier S. 201f.

3 J. Kocka, Siemens und der aufhaltsame Aufstieg der AEG, in: Tradition 17, 1972, S. 125-142. 
den. ${ }^{4}$ Mit dieser Aufgabe wurde der 27jährige Diplomingenieur Wichard von Moellendorff betraut. Er erhielt damit eine Schlüsselposition beim Auf- und Ausbau der wissenschaftlichen Strukturen der AEG.

Moellendorff entstammte einer Familie mit naturwissenschaftlicher Tradition. Sein Vater, promovierter Zoologe, hatte neben seiner Amtstätigkeit als deutscher Konsul in Manila die Molluskenfauna der Philippinen erforscht und beschrieben. Seine Mutter war Tochter des in Südosteuropa tätigen deutschen Konsuls, Orientalisten und Botanikers Otto Blau. Moellendorff studierte Maschinenbau an der Technischen Hochschule Charlottenburg. Sein materialkundliches Interesse wurde hier sicherlich durch Adolf Martens ${ }^{5}$ geweckt, den Vater der wissenschaftlichen Werkstoffprüfung, der viele Prüfmethoden und -maschinen entwickelt hatte, erster Direktor des Königlichen Materialprüfungsamtes war und dem zu Ehren die kohlenstoffreiche Gefügeform des gehärteten Stahls Martensit heißt.

Neben dem Studium begeisterte sich Wichard von Moellendorff für den zeitkritischen Journalisten Maximilian Harden und dessen Zeitschrift Die Zukunft und trat mit Harden in Kontakt. Harden machte seinen jungen Anhänger u.a. mit dem ihm befreundeten Walther Rathenau bekannt, dem Sohn des AEG-Gründers und späteren Außenminister. Diese Begegnung wurde entscheidend für Moellendorffs Weg. Rathenau, Vorstandsmitglied (später Präsident) der AEG, erkannte und schätzte die Begabungen des strebsamen jungen Mannes und stellte ihn nach Ablegung des Diplomexamens 1906 zunächst als konstruierenden Maschinenbauingenieur im Kabelwerk Oberspree ein. Moellendorff wurde zum Bewunderer des 14 Jahre älteren, brillanten, universell gebildeten Walther Rathenau, dessen Bücher er 1913 einmal als seinen „Katechismus“" bezeichnete.

Sein Metall-Laboratorium gliederte Moellendorff in drei „Stationen: eine mechanische, eine chemische und eine mikroskopische“, die „teils zentralisierte Kontrolle, teils Forschungsarbeiten erledigen" sollten. ${ }^{8}$ Neben der Leitung des Laboratoriums ${ }^{9}$ und eigener wissenschaftlicher Sacharbeit dort wurde

4 W. von Moellendorff, Die Wechselbeziehung zwischen der empirischen Metalltechnik und der Metallographie, in: Giesserei-Zeitung 11, 1914, S. 506-509 u. S. 512-525, hier S. 512.

5 A. Martens, Handbuch der Materialienkunde für den Maschinenbau, Erster Theil, Materialprüfungswesen, Probirmaschinen und Messinstrumente, Berlin 1898 u. ders., Handbuch der Materialienkunde für den Maschinenbau, Zweiter Teil, Die technisch wichtigsten Eigenschaften der Metalle und Legierungen von E. Heyn. Hälfte A, Die wissenschaftlichen Grundlagen für das Studium der Metalle und Legierungen. Metallographie, Berlin 1912.

6 Biografie: Wichard von Moellendorff, 1881-1937; Deutsches Historisches Museum Berlin, Lebendiges Virtuelles Museum Online (LeMo). http./www.dhm.de/lemo/html/biografien/ MoellendorffWichard/.

7 W. von Moellendorff, Das technische Motiv, in: Die Zukunft 85, 1913, S. 419.

8 Moellendorff (wie Anm. 4), S. 512.

9 Näheres über die personelle, apparative und finanzielle Ausstattung des Laboratoriums, sein Arbeitsprogramm und insbesondere den Bereich der Materialprüfung außerhalb der 
Moellendorff dank seinem Organisationstalent und Blick für das Ganze bald Direktionsassistent und Leiter der technisch-ökonomischen Verwaltungsabteilung des Kabelwerks Oberspree.

\section{Materialprüfung und Metallforschung bei der AEG unter Moellendorff}

Moellendorff etablierte in seinem Prüflaboratorium als Ergänzung zur chemischen Analyse die metallographische Analyse: sie sei „billig, zuverlässig und äußerst sinnfällig“ und gestatte, „den Aufbau der Stoffe direkt zu beobachten und seine Abhängigkeit von chemischen, thermischen und mechanischen Einflüssen im Bilde zu verfolgen. Wie einst das Mikroskop die Kunde von den lebenden Körpern in ungeahntem Grade bereicherte, so verheißt es auch der Technik und ihrer Materialkunde eminenten Nutzen." ${ }^{10}$ Der Hinweis auf das Mikroskop verdeutlicht, dass die Zeit des „Versuchs und Irrtums" auch in der Metalltechnik zu Ende gekommen war. Nennenswerte Verbesserungen der Produkte erforderten die Einbeziehung wissenschaftlicher Methoden. Die wissenschaftlich-theoretisch geleitete Arbeit hielt ihren Einzug in die Metalltechnik.

Moellendorff zeigte, dass die metallographische Analyse „eine vorzügliche Methode“ ist, das in einer ,großen elektrotechnischen Fabrik verwendete Kupfer auf seine Reinheit zu prüfen" ${ }^{11}$ wie es die Abbildungen 2(a) und (b) aus dem Jahr 1910 mit Fotos von Schliffen von zwei Kupferproben verdeutlichen. ${ }^{12}$ Abbildung 2a zeigt eine erstarrte Kupferschmelze, die überhitzt wurde. Dabei hat das Kupfer große Mengen Sauerstoff aufgenommen und Kupferoxid $\mathrm{Cu}_{2} \mathrm{O}$ gebildet. Durch Ausmessen der Flächen der verschiedenen Phasen im Schliffbild ließ sich der Sauerstoff semiquantitativ analysieren. Abbildung $2 b$ stellt eine erstarrte zweimal reduzierte Kupferschmelze dar. ,Jetzt sind selbst Spuren von Oxydul im Bild nicht mehr zu finden“. Den damaligen Verfahrensstand der Kupferraffination verdeutlicht die folgende Beschreibung: „Die Reduktion bewirkt man durch Eintauchen eines Birkenstammes, dessen Bestandteile, Kohlenstoff und Wasserstoff, zum Sauerstoff eine so starke chemische Affinität besitzen, dass der Sauerstoff ganz plötzlich in großen Mengen das Kupfer verlässt und sich unter heftiger Wallung der Schmelze den reduzierenden Elementen verbindet. ${ }^{\text {"13 }}$

Metallographie ist nicht bekannt, da Akten des Kabelwerks Oberspree aus jener Zeit fehlen. Unsere Darstellung muss sich auf die technisch-wissenschaftlichen Veröffentlichungen stützen.

10 W. von Moellendorff, Metallgefüge, in: AEG-Zeitung 13, 1910, H. 4, S. 9-11, hier S. 11.

11 Wissenschaftliche Materialprüfung bei der AEG, in: AEG-Zeitung 15, 1913, H. 11, S. 2 8, hier S. 8 (ohne Autorenangabe, wahrscheinlich von W. von Moellendorff).

12 Moellendorff (wie Anm. 10), S. 10f.

13 Ebd., S. 11. 

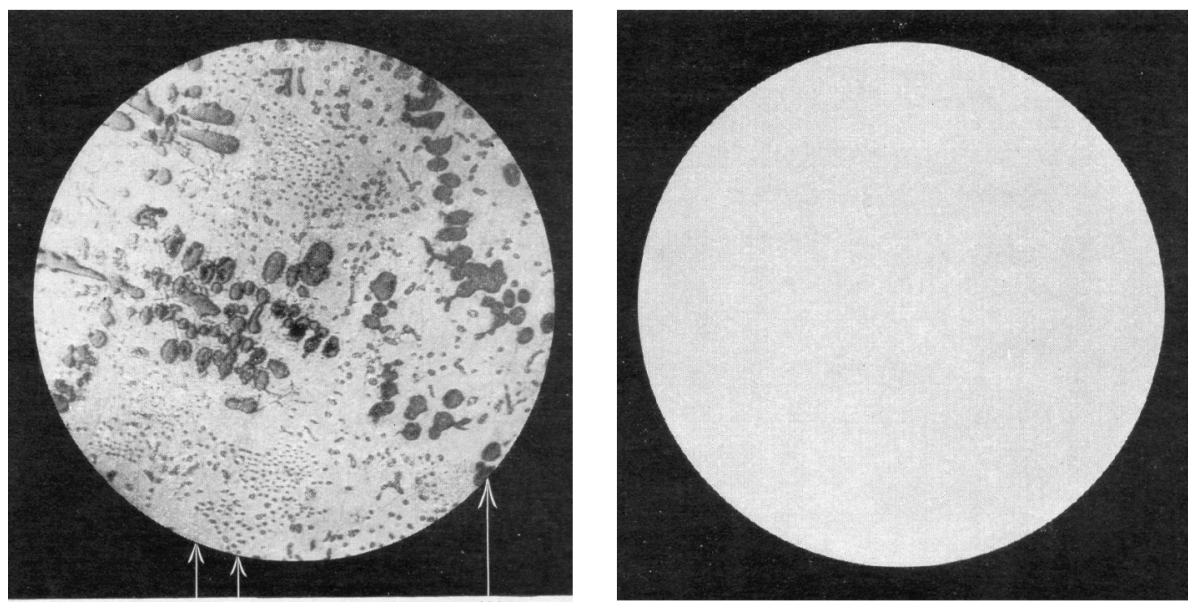

(1) (2)

(3)

Abb. 2: Schliffe von erstarrten Kupferschmelzen: (a) erstarrte Kupferschmelze, die überhitzt wurde; (1): reines $\mathrm{Cu},(2): \mathrm{Cu} / \mathrm{Cu}_{2} \mathrm{O},(3)$ : reines $\mathrm{Cu}_{2} \mathrm{O}$. (b) erstarrte völlig gereinigte Kupferschmelze. (Quelle: AEG-Zeitung 13, 1910, H. 4, S. 10)

Reinheit und zugleich Kosten der Materialien wurden zunehmend wichtig. Moellendorff stellte 1913 fest: ${ }^{14}$ „Die technologische Fertigkeit, Kupferbarren von gleichzeitig völliger Reinheit und genügender Dichte zu gießen, also das zur Drahtfabrikation nötige Vorprodukt zu erzeugen, ist ganz jungen Datums. Noch vor 20 Jahren war Legieren und damit Minderung der Leitfähigkeit der einzige Weg, auf dem man (Kupfer-)Barren zur Drahtfabrikation herstellen konnte“. Beim ,heutigen Elektrolytkupfer mit seinem normalen Reingehalt von mehr als 99,9\% ist trotzdem der Kupferpreis im Laufe des vorigen Jahrhunderts auf etwa die Hälfte gesunken.“

Zunehmende Massenherstellung, Export und Weiterverwendung für immer unterschiedlichere Anwendungen machten Gleichförmigkeit und Standardisierung der Produkte dringend notwendig. Moellendorff betonte den Bedarf nach Normung technischer Legierungen. 1914 ,deckte die AEG ihren Weltmarkt mit nur sieben walz- und ziehbaren Bronzen“, ${ }^{15}$ deren Gehalt an Kupfer, Zinn, Zink und Blei eindeutig definiert war, während es weltweit „schätzungsweise 500 technische Bronzesorten“ gab. Diese stellten nach Moellendorff kein „Reichtums-, sondern ein Armutszeugnis der Legierungskunde“ dar, „so wie die deutsche Landkarte des 18. Jahrhunderts das Symbol der politischen Schwäche war“".

14 W. von Moellendorff, Über das Kabelwerk Oberspree. Vortrag mit kinematographischer Vorführung am 4. Februar 1913 in Wien, in: Elektrotechnik und Maschinenbau 31, 1913, S. 242-244, hier S. 243.

15 W. von Moellendorff, Nomenklatur und Normalien für Bronze, in: Giesserei-Zeitung 11, 1914, S. 26-28, hier S. 28. 
Den Erfolg seines AEG-Labors für das Unternehmen hat Moellendorff später einmal so beschrieben: ${ }^{16}$ „Das größte deutsche Metallwerk stellte sich von der Empirie zur Systematik um. Mir fiel das schöne Los des Patrouillenreiters zu. ... Das dicke Buch chemischer und physikalischer Geheim- und Zufallsrezepturen schrumpfte zu einem schmalen Heft zusammen, das eine planmäßige, stetige Fabrikation gewährleistete. Der Ballast des Abfall- und Ausschussmaterials verkleinerte sich“. Ähnlich wie 40 Jahre früher die chemische Industrie mit der Aufstellung der ringförmigen Benzolformel durch Kekulé und der nachfolgenden Isolierung und Synthetisierung vieler Benzolderivate ging jetzt die Metalltechnik den Weg der systematischen Erforschung technisch wichtiger Metalle und Legierungen und der Nutzung der Ergebnisse. 1913 nannte Moellendorff seinen Arbeitsbereich „wissenschaftliche Materialprüfung bei der AEG“. ${ }^{17}$

Neben der Materialprüfung und der angewandten Metallforschung führte das Labor auch kristallographisch-technologische Grundlagenarbeiten durch. Moellendorff und sein Assistent Czochralski, der an der Technischen Hochschule Charlottenburg Chemie studiert hatte, veröffentlichten 1913 die Arbeit Technologische Schlüsse aus der Kristallographie der Metalle. ${ }^{18}$ Sie hatten Wachstumskristalle von Blei, Wismut, Zink, Aluminium, Kupfer, Messing und Zinn-Legierungen hinsichtlich Erstarrungsoberflächen, Korngestalt, Bruchflächen und Korngröße untersucht. Dabei beobachteten sie an Kupfer Zwillingsbildung und brachten Blei ,mit den Umrissen der dichtesten Kugelpackung zum Erstarren“. Sie interessierten sich für das „Wesen des Warmbruches" an vielen technisch wichtigen Metallen wie z.B. Kupfer, Bronze, Messing, Aluminium, Zink und Zinn. Weiterhin untersuchten sie metallographische Metalle auch vor und nach „beträchtlicher Kaltreckung“, d.h. plastischer Verformung durch starkes Ziehen unterhalb der Rekristallisationstemperatur. Sie stellten fest, dass beim Kaltrecken Kristallite verbogen und in den ,Zustand einer starken inneren elastischen Verspannung versetzt werden, jedoch, jeder für sich betrachtet, als ein ganzes Individuum bleibt.“ „Erst beim Glühen, d.i. oberhalb der dem Material mit seinem Bearbeitungszustand zugehörigen Rekristallisationstemperatur, erlangen die Moleküle soviel Beweglichkeit, dass aus der vorangegangenen gewaltsamen Verlagerung willkürliche neue kristallinische Orientierung, wahrscheinlich oft in Form von Zwillingen, hervorgeht." Mit diesen Ergebnissen widerlegten sie den Altmeister der deutschen Metallkunde, Gustav Tammann in Göttingen. Faust und Tammann hatten angenommen, dass beim Kaltrecken die Zahl der Kristallite („Kornzahl“) mit zunehmendem Fließdruck propor-

16 W. von Moellendorff, Konservativer Sozialismus, Hamburg 1932, S. 56.

17 Wissenschaftliche Materialprüfung (wie Anm. 11), S. 2.

18 W. von Moellendorff u. J. Czochralski, Technologische Schlüsse aus der Kristallographie der Metalle, in: Zeitschrift des Vereins Deutscher Ingenieure 57, 1913, S. 931-935 u. 1014-1020. 
tional steige und dann bei der oberen Elastizitätsgrenze konstant werde. ${ }^{19}$ Die AEG-Forscher fanden dagegen: „Kornverfeinerung durch eigentliches Kaltrecken tritt erst ein, wenn zugleich die Formbarkeit des Metalles erschöpft, das Arbeitsgut also bis zum Bruch angestrengt und verfestigt ist“. „So lassen die jüngsten einschlägigen Aufsätze von Faust und Tammann" die bisherigen „Theorien beiseite, obgleich mindestens der Versuch ihrer Entkräftung gelohnt hätte, ehe man neue Hypothesen aufstellte.“20 „Meines Erachtens spricht für Tammanns Auffassung keine einzige Experimentalbeobachtung“".

Ihre Deutung der Verfestigung beim Kaltrecken, die nach der Tammannschen Theorie nicht zu verstehen ist und die damals eine der wichtigsten Fragen der Metalltechnik war, haben Moellendorff und Czochralski auch durch Verfahren der Materialprüfung belegt. Sie werteten Zerreißversuche an kaltgereckten zylindrischen Proben als Spannungs-Verformungs-Diagramme auf eine neue Art aus, indem sie effektive Zugspannungen $s$ in $\mathrm{kg} \mathrm{mm}^{-2}$ als Funktion der relativen Querschnittsverringerung $(-\Delta Q / Q) \times 100$ in Prozent auftrugen. Die 1913 publizierten Diagramme ${ }^{22}$ für verschieden vorbehandelten Kupferdraht bei Zimmertemperatur und einer „Reckgeschwindigkeit" von 20 „Atmosphären pro Minute" sind in Abbildung 3 (S. 146) gezeigt.

Im Gegensatz zur üblichen Darstellung der Zugspannung als Funktion der Dehnung, bei der die Zugspannung ein Maximum durchläuft und dann vor dem Zerreißen wieder abfällt, steigt in der Darstellung von Moellendorff und Czochralski die effektive Zugspannung monoton an. Bei der plastischen Formänderung zwischen Streckgrenze und Höchstlastgrenze wird der Probestab auf seiner ganzen Länge zwar gleichmäßig gering verjüngt, aber dabei stark verfestigt. Nach Ansicht der beiden AEG-Forscher erfolgt hier „bleibende, wenn auch winzige Molekularbewegung (Drehung)“. Im Bereich jenseits der Höchstlastgrenze bis zum Zerreißen entstehen weitere plastische, aber jetzt größere, lokalisierte Formänderungen (Fließkegel) durch „,vorwiegend Gleitungen“. Die Kurven verlaufen bei Überschreitung der Höchstlastgrenze zunächst geradlinig, und die Verlängerungen dieser Geraden schneiden sich in einem ,gemeinsamen virtuellen Endpunkt" beim Querschnitt Null. Mit den allgemeinen Koordinaten Zugspannung $s$, Querschnitt $q$ an der engsten Stelle und den Koordinaten der Höchstlastgrenze $s_{2}, q_{2}$ gilt $\left(s-s_{2}\right) / s_{2}=\left(q_{2}-q\right) / q_{2}$. Die Zugspannung bei der Höchstlastgrenze ist gerade halb so groß wie die im virtuellen Endpunkt, $s_{2}=s_{4} / 2$ (Abb. 3), wie sich auch durch Einsetzen des Querschnitts

19 O. Faust u. G. Tammann, Eine Methode zur Bestimmung der untern und obern Elastizitätsgrenze und über die Verfestigung der Metalle, in: Zeitschrift für Physikalische Chemie 75, 1911, S. 108-126.

20 Moellendorff/Czochralski (wie Anm. 18), S. 1014.

21 W. von Moellendorff, Bemerkungen gegen den Amorphismus der Metalle, in: Internationale Zeitschrift für Metallographie 6, 1914, S. 44-47, hier S. 47.

22 Moellendorff/Czochralski (wie Anm. 18), S. 1018. 


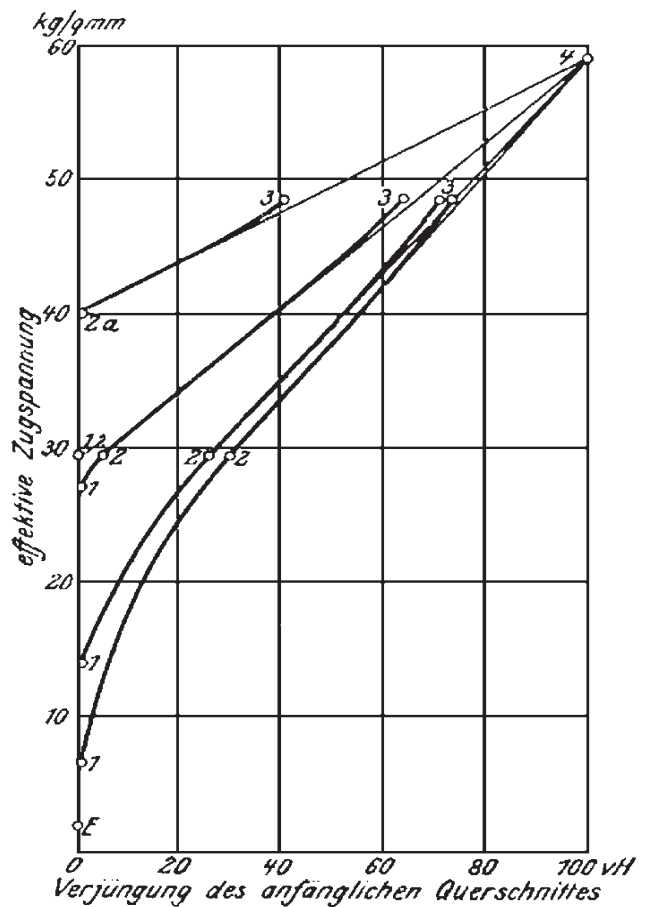

Abb. 3: Spannungs-Verformungskurven für geglühten, verschieden stark vorgereckten Kupferdraht bei Zimmertemperatur und bei einer „Reckgeschwindigkeit" von 20 „Atmosphären pro Minute“. Aufgetragen ist die effektive Zugspannung in $\mathrm{kg} \mathrm{mm}^{-2}$ als Funktion der Abnahme des anfänglichen Querschnittes in Prozent. (1) Streckgrenze, (2) Höchstlastgrenze, (3) Bruchgrenze, (4) gemeinsamer „virtueller Endpunkt“ aller Kurven. (Quelle: Zeitschrift des Vereins Deutscher Ingenieure 57, 1913, S. 1018)

$q\left(s_{4}\right)=0$ ergibt. Die obige allgemeine Gesetzmäßigkeit wurde 16 Jahre später auch theoretisch begründet. ${ }^{23}$ Noch etwa 20 weitere Jahre intensiver Forschungsarbeit auf dem technologisch so wichtigen Gebiet der Metallverfestigung durch Kaltbearbeitung waren jedoch nötig, bis ein völlig neues Modell die richtige atomare Deutung der Vorgänge ermöglichte. Dies zeigt zweierlei: zum einen, dass für die industrielle Verwertung ein zufrieden stellendes praktisches Ergebnis ohne vollständige theoretische Grundlagen ausreichte, zum anderen aber, dass Fragen der praktischen Anwendung oftmals den Anstoß für lange Jahre der grundlagennahen theoretischen Forschung gaben.

Die Weiterentwicklung der Grundlagenarbeiten über Metallkristalle führte dann, als Moellendorff bereits in die Politik übergewechselt war, zur aus heutiger Sicht größten Entdeckung, die im AEG-Metall-Laboratorium gemacht wurde: dem Einkristallziehen aus einem mit einer Schmelze gefüllten Tiegel. Abbildung 4 zeigt ein Foto der chemischen „Station“ des MetallLaboratoriums von 1911. Nach Recherchen von Tomaszewski ${ }^{24}$ handelt es

23 E. Schiebold u. G. Richter, Mitteilungen aus den deutschen Materialprüfungsanstalten 1929, Sonderheft V: Arbeiten aus dem Materialprüfungsamt und dem Kaiser-WilhelmInstitut für Metallforschung zu Berlin-Dahlem, S. 68-96, hier S. 84.

24 P. E. Tomaszewski, private Mitteilung 2003. 
sich bei dem im Vordergrund links am Schreibtisch sitzenden Wissenschaftler um den Chemiker Jan Czochralski, der von 1911 bis 1914 Moellendorffs Assistent war und danach die Leitung des Laboratoriums übernahm. Bei einem missglückten Experiment, die Kristallisationsgeschwindigkeit von Zinn zu messen, gelang ihm 1916 durch Zufall diese herausragende Entdeckung. ${ }^{25}$ Nach dem Czochralski-Verfahren werden heute 95\% der in der HalbleiterMikroelektronik benötigten Siliciumeinkristalle produziert! ${ }^{26}$

\section{Nationalökonom und Politiker}

Mit Beginn des Ersten Weltkrieges im August 1914 gefährdete die alliierte Blockade die Versorgung Deutschlands mit Nahrungsmitteln, Munition, chemischen Grundstoffen und Buntmetallen. Moellendorff, mit Buntmetallen täglich beschäftigt, erkannte dies früh ${ }^{27}$ und schlug Walther Rathenau vor, eine zentrale staatliche Bewirtschaftung der wichtigsten Rohstoffe anzure-

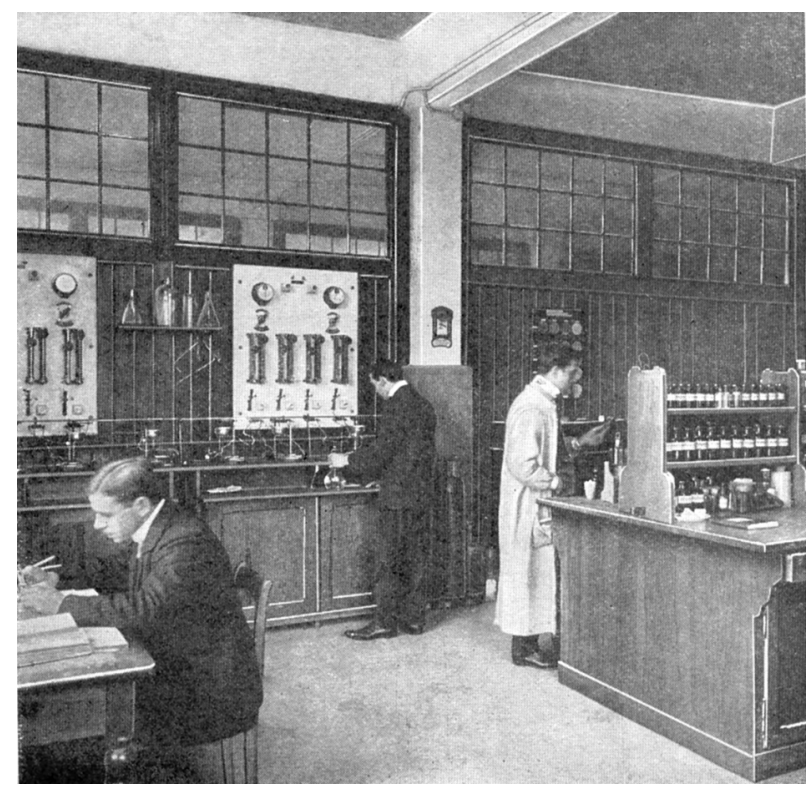

Abb. 4: Blick in die chemische „Station“ des Metall-Laboratoriums Oberspree. Links im Vordergrund Jan Czochralski, der spätere Entdecker des Einkristallziehens aus der Schmelze. (Quelle: AEG-Zeitung 13, 1911, H. 9, S. 14)

25 J. Czochralski, Ein neues Verfahren zur Messung der Kristallisationsgeschwindigkeit der Metalle, in: Zeitschrift für Physikalische Chemie 92, 1918, S. 219-221.

26 J. Evers, P. Klüfers, R. Staudigl u. P. Stallhofer, Czochralskis schöpferischer Fehlgriff: ein Meilenstein auf dem Weg in die Gigabit-Ära, in: Angewandte Chemie 115, 2003, S. 5862-5877 und Angewandte Chemie 42, 2003, S. 5684-5698.

27 K. Braun, Konservatismus und Gemeinwirtschaft, Eine Studie über Wichard von Moellendorff, Duisburg 1978, S. 53. 


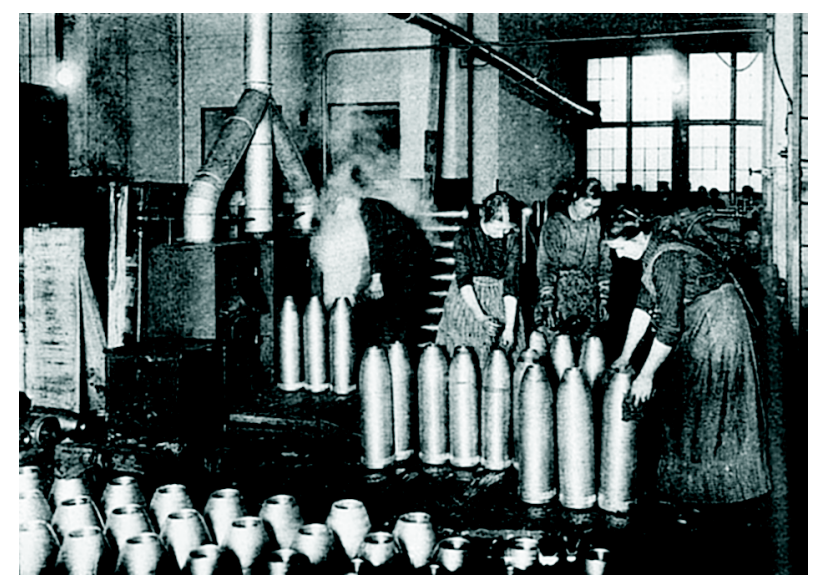

Abb. 5: Wurfminenhülsen produziert bei der AEG in Berlin: Reinigen durch einen Arbeiter und Lackieren durch drei Arbeiterinnen. (Quelle: L. Kugler, Die AEG im Bild, Berlin 2001, S. 112, mit freundlicher Genehmigung der Nicolaischen Verlagsbuchhandlung Beuermann GmbH)

gen. Rathenau erwirkte im Kriegsministerium die Gründung der Kriegsrohstoffabteilung, ${ }^{28}$ wurde ihr Leiter und stellte Moellendorff dort als einen der ersten Referenten ein. ${ }^{29}$ Die AEG entwickelte sich unterdessen mit Beginn des Weltkrieges schnell zum „,zweitgrößten Rüstungslieferanten Deutschlands nach Krupp“. ${ }^{30}$

Moellendorffs Tätigkeit in der Kriegsrohstoffabteilung brachte ihn u.a. in engen Kontakt mit dem Direktor des Kaiser-Wilhelm-Instituts für physikalische Chemie und Elektrochemie, dem späteren Nobelpreisträger Fritz Haber, ${ }^{31}$ der das Ministerium in chemischen Fragen beriet. Moellendorff stieg auch im neuen Wirkungskreis schnell auf: 1915 wurde er zum Direktor der Kriegschemikalien AG ernannt, 1916 zum Reichskommissar für Kalkstickstoff. Neben der Tagesarbeit konzipierte und beschrieb er eine „Deutsche Gemeinwirtschaft", ${ }^{32}$ ein Kompromissmodell zwischen kapitalistischer Markt- und sozialistischer Planwirtschaft.

Im April 1918 wurde Moellendorff auf Empfehlung von Fritz Haber auf eine ordentliche Professur für Nationalökonomie und Finanzwissenschaften

28 Biografie: Walther Rathenau, 1867-1922; Deutsches Historisches Museum Berlin; Lebendiges Virtuelles Museum Online (LeMo). htttp:/www.dhm.de/lemo/html/biografien/ RathenauWalther/.

29 Braun (wie Anm. 27), S. 51.

30 L. Kugler, Die AEG im Bild, Berlin, 2001, S. 112.

31 Biografie: Fritz Haber, 1868-1934; Deutsches Historisches Museum Berlin. Lebendiges Virtuelles Museum Online (LeMo). http://www.dhm.de/lemo/html/biografien/HaberFritz/ index.html; - R. Hoffmann u. P. Laszlo, Fritz Habers düsterer literarischer Schatten, in: Angewandte Chemie 113, 2001, S. 4733-4739, und Angewandte Chemie (Int. Ed.) 40, 2001, S. 4599-4604.

32 W. von Moellendorff, Deutsche Gemeinwirtschaft, Berlin 1916. 
an die TH Hannover berufen. Die Lehrtätigkeit blieb jedoch Episode, denn schon im November des gleichen Jahres, nach Kriegsende, Revolution und Zusammenbruch der Monarchie, holte der sozialdemokratische Minister Wissell Moellendorff als Unterstaatssekretär ins Reichswirtschaftsamt, um mit ihm die gemeinwirtschaftlichen Vorstellungen im neuen Staat zu verwirklichen. Die Vorschläge zu einer gemäßigten Sozialisierung im Montanbereich und einer Wirtschaftssteuerung durch ein System von Räten und Fachgremien stießen auf ein geteiltes Echo. Anfang Dezember 1918 nahm Haber in einem Brief an Moellendorff zu dessen Bemühungen sympathisierend, aber skeptisch hinsichtlich der Durchsetzbarkeit Stellung: „Die Revolution war ein Femegericht über das Regierungssystem, nicht über den Capitalismus. Die Nation ist ,bourgeois' bis in die Knochen. ${ }^{\text {"33 }}$ In der entscheidenden Kabinettssitzung 1919 stimmte kein anderer Minister dem von Wissell vorgetragenen Grundsatzplan zu; den Bürgerlichen ging er viel zu weit, den Sozialdemokraten nicht weit genug. Wissell und Moellendorff traten von ihren Ämtern zurück.

Das Verhältnis zu Walther Rathenau kühlte sich nach der Revolution ab. Rathenau beanspruchte öffentlich die Urheberschaft am Gemeinwirtschaftsgedanken für sich. Moellendorff hielt ihm unter anderem entgegen, dass er, Rathenau, selbst nach wie vor einer rein kapitalistischen Wirtschaftsweise anhänge. Ein in der Presse ausgetragener Prioritätenstreit darum, wer von beiden ursprünglich 1914 die Kriegs-Rohstoffbewirtschaftung vorgeschlagen habe, beendete schließlich 1920 die enge Beziehung der beiden Männer.

Moellendorffs ökonomische Sachkenntnis und Erfahrung blieben auch nach seinem politischen Scheitern weiterhin gefragt. Neben verschiedenen Aufsichtsratsmandaten arbeitete er ab 1926 im „Gemischten wirtschaftlichen Unterausschuss der vorbereitenden Abrüstungskonferenz" in Genf mit. ${ }^{34}$

Auch als Wirtschafts- und Gesellschaftstheoretiker blieb Moellendorff geistig zeitlebens der Ingenieurwissenschaftler, der, wo immer möglich, Beobachtung und Messung an die Stelle von Vermutung und Spekulation zu setzen suchte. Er benutzte den Begriff des Wirkungsgrades, um den relativen Nutzen ökonomischer und politischer Maßnahmen zu bewerten. Um eine Datenbasis für wirtschaftspolitische Überlegungen zu schaffen, verfasste er auf der Grundlage eigener umfangreicher quantitativ-empirischer Erhebun-

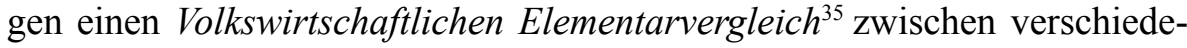

33 F. Stern, Freunde im Widerspruch. Haber und Einstein, in: R. Vierhaus u. B. vom Brocke (Hg.), Forschung im Spannungsfeld von Politik und Gesellschaft: Geschichte und Struktur der Kaiser-Wilhelm-/Max-Planck-Gesellschaft, Stuttgart 1990, S. 516-551, hier S. 533.

34 Braun (wie Anm. 27), S. 174.

35 W. von Moellendorff, Volkswirtschaftlicher Elementarvergleich zwischen den Vereinigten Staaten von Amerika, Deutschland, Großbritannien, Frankreich und Italien. Entwurf besprochen von A. Reithinger, in: Naturwissenschaften 19, 1931, S. 226-230. 
nen Ländern. Teile aus seinen wirtschafts- und gesellschaftspolitischen Schriften wurden später in dem Sammelband Konservativer Sozialismus ${ }^{36}$ zusammengefasst. Moellendorffs volkswirtschaftliches und politisches Wirken ist in zwei Dissertationen dargestellt und diskutiert worden. ${ }^{37}$ Bis heute gelten seine Ideen und Bestrebungen manchen als zu planwirtschaftlich und marktfern; ihm als „Planungsenthusiasten“ wurde sogar das Fortführen der kriegsbedingten Planwirtschaft in die Friedenszeit als Unfähigkeit vorgeworfen, den neuen Staat lebensfähig zu gestalten. ${ }^{38}$ Andere haben gerade in der Abkehr von einer reinen Marktwirtschaft ein Ernstnehmen der Revolution von 1918 gesehen. $^{39}$

\section{Kaiser-Wilhelm-Institut für Metallforschung und Staatliches Material- prüfungsamt unter Moellendorff}

Die Kaiser-Wilhelm-Gesellschaft zur Förderung der Wissenschaften (KWG) hatte nach ihrer Gründung im Jahr 1911 bis zum ersten Kriegsjahr neun Institute errichtet. Noch während des Krieges wurde ab 1917 ein neues Institut für Metallforschung geplant. Ideen und Gutachten dazu wurden von vier Persönlichkeiten erbeten: von den Stahl-Großindustriellen Gustav Krupp und Hugo Stinnes, von Richard Merton, dem späteren Leiter der Metallgesellschaft, aber auch von Wichard von Moellendorff. ${ }^{40}$ Im Juli 1920 wurde in Neubabelsberg bei Berlin in gemieteten Laboratorien und Hallen einer ehemaligen Munitionsfirma das Kaiser-Wilhelm-Institut (KWI) für Metallforschung gegründet. Sein Direktor war Adolf Martens' ehemaliger Mitarbeiter Emil Heyn. Heyn verstarb jedoch schon im März 1922. Die Suche nach einem neuen Leiter erwies sich als schwierig, u.a. wegen der unsicheren wirtschaftlichen Lage des Instituts. Moellendorff wurde zum Nachfolger vorgeschlagen, aber Fritz Haber opponierte jetzt heftig gegen ihn; Moellendorff, der Ingenieur, sei kein Gelehrter auf einem Spezialgebiet und werde eine zu industrienahe, technisch ausgerichtete Forschung etablieren, anstatt selbstständig naturwissenschaftliche Grundlagen der Metallkunde zu erforschen.

Unterdessen wurde Moellendorff am 1. April 1923 vom preußischen Kultusminister - zunächst kommissarisch - auf die frei gewordene Stelle des Direktors des Staatlichen Materialprüfungsamtes berufen. ${ }^{41}$ Etwa zur

36 Moellendorff (wie Anm. 16).

37 Braun (wie Anm. 27) und Dieter Schmid, Wichard von Moellendorff, Ein Beitrag zur Idee der wirtschaftlichen Selbstverwaltung, Diss. FU Berlin, 1970.

38 G.D. Feldman, The Great Disorder: Politics, Economics, and Society in the German Inflation, 1914-1924, New York 1993.

39 O.H. von der Gablentz, Zu Unrecht vergessen: Wichard von Moellendorff, in: Gewerkschaftliche Monatshefte 5, 1954, S. 362-364.

40 Marsch (wie Anm. 1), S. 392.

$41 \mathrm{Ob}$ es hierzu durch eigene Initiative Moellendorffs oder durch Anregung von dritter Seite kam, ist nicht bekannt. 
selben Zeit setzte die dramatische Phase der Inflation ein und entwertete die von der Industrie für das junge KWI bereitgestellten Gelder völlig, so dass dessen Weiterarbeit akut gefährdet war. Moellendorff hatte jetzt die Möglichkeit, das Institut in die Gebäude des Materialprüfungsamtes in BerlinDahlem, dem ,deutschen Oxford“, mit aufzunehmen und ihm dadurch Mietkosten zu ersparen. Übernahm Moellendorff dazu in Personalunion die Institutsleitung, konnten Kompetenzüberschneidungen vermieden und auch noch ein Direktorengehalt eingespart werden. Die KWG entschied sich für diesen Weg. Ende 1923 waren beide Einrichtungen im Dahlemer Gebäudekomplex vereinigt. Die analytische Abteilung des KWI wurde aufgelöst, da eine entsprechende Abteilung im Materialprüfungsamt bestand. Ebenso wurde die metallurgische Abteilung geschlossen, da ihr bisheriger Leiter auf eine Hochschulprofessur wechselte; Moellendorff hatte die Metallurgie, also Fragen der Metallgewinnung aus Erzen, als Arbeitsgebiet des Instituts von Anfang an abgelehnt. Übernommen wurden die metallographische und die (erst geplante) Röntgenabteilung. Die kostensparende „Symbiose“ von Materialprüfungsamt und KWI sicherte für die folgenden Jahre die Weiterexistenz des Metallinstituts und ermöglichte es, die Röntgenabteilung des Instituts unter ihrem Leiter Ernst Schiebold aufzubauen..$^{42}$ Moellendorff sah seine persönliche Hauptaufgabe in der Koordination beider Einrichtungen sowie für das Materialprüfungsamt in einer Neuorganisation und in der Angleichung der Mitarbeiterbesoldung an diejenige anderer, vergleichbarer Reichs- und preuBischer Anstalten. Die letzteren Bemühungen waren nur teilweise erfolgreich. ${ }^{43}$ Tagesfragen der wissenschaftlichen Leitung des KWI überließ Moellendorff meist seinem Stellvertreter Oskar Bauer.

Die enge Verbindung der beiden Einrichtungen entsprach über die Kostenersparnis hinaus Moellendorffs ganzheitlichem Denken: „Die Gemeinschaft des Amtes mit dem Metallforschungsinstitut bewährt sich gut. ... Auch die rein geistige Berührung zwischen der empirisch-statistisch eingestellten Technologie und der exakten Physikochemie erweist sich als fruchtbar. Ähnliche Gemeinschaften auf anderen Gebieten wären durchaus zu begrüßen." ${ }^{\text {"44 Auch }}$ die beiden Zeitschriften Mitteilungen aus dem Materialprüfungsamt und Mitteilungen aus dem KWI für Metallforschung wurden 1923 vereinigt.

42 Marsch (wie Anm. 1), S. 403.

43 W. Ruske, G.W. Becker u. H. Czichos, 125 Jahre Forschung und Entwicklung, Prüfung, Analyse, Zulassung, Beratung und Information in Chemie und Materialtechnik, Bundesanstalt für Materialforschung und -prüfung (BAM), Berlin, Bremerhaven 1996, S. 135 und 138f. (Der dort angegebene Name ,von Moellendorff-Wilamowitz“ ist unrichtig).

44 Mitteilungen aus dem Materialprüfungsamt und dem Kaiser-Wilhelm-Institut für Metallforschung zu Berlin-Dahlem 42, 1924, S. 1-4, hier S. 1. 
Moellendorff selbst untersuchte 1923 die Gestalt des Fließkegels ${ }^{45}$ der sich beim Zugversuch in zylindrischen Proben im Gebiet zwischen Höchstlastgrenze und Bruchgrenze (s. Abb. 3) bildet, eine Weiterführung der 1913 bei der AEG begonnenen Untersuchungen. Er zeigte, dass die Mantellinien der Fließkegel in so unterschiedlichen Materialien wie „mittelharter Stahl, weiches Flusseisen, weiches Kupfer, Blei und Plastilin“ eine „geometrisch ähnliche Kontur" aufweisen und mit einer einfachen logarithmischen Formel beschreibbar sind. Durch Einfügen einer Proportionalitätskonstante modifiziert wurde die logarithmische Formel sechs Jahre später von Schiebold und Richter theoretisch abgeleitet. ${ }^{46}$ Unter Moellendorffs Amts- und Institutsleitung erschienen von 1926 bis 1929 in den Mitteilungen aus dem Materialprüfungsamt und dem KWI für Metallforschung noch etwa 20 Untersuchungen zum Thema Festigkeit, Spannungen und Härte von Kristallen, u.a. von später weltbekannten Forschern wie Max Hansen, Ernst Schiebold und Erich Schmid. Die experimentellen Möglichkeiten der Röntgenstrukturanalyse im Institut waren inzwischen weiter verbessert worden. ${ }^{47}$ Außerdem erfolgte eine intensive Zusammenarbeit mit Forschern anderer Dahlemer KWI wie Karl Weißenberg (Institut für Physik) und Michael Polanyi (anfänglich Institut für Faserforschung, später Institut für physikalische Chemie und Elektrochemie). Weißenberg schreibt im Sonderheft III der Mitteilungen 1927 über Kristallaufbau und chemische Konstitution, ${ }^{48}$ während in Sonderheft II von 1926 Polanyi zusammen mit Georg Sachs vom Metallforschungsinstitut, ${ }^{49}$ der von 1926 bis 1930 das Röntgenlaboratorium weiter ausbaute, über ,innere Spannungen“" an Kristallen berichtet.

Der wissenschaftliche Durchbruch auf dem Gebiet der kalt bearbeiteten Metalle gelang 1932 Michael Polanyi, der zusammen mit Henry Eyring auch die moderne chemische Reaktionskinetik begründete. Daneben war sein spezielles Forschungsgebiet die Röntgenstrukturanalyse kalt bearbeiteter Metalle, wo er mit Georg Sachs und ganz besonders mit Erich Schmid vom

45 W. von Moellendorff, Die Gestalt des Fließkegels, in: Mitteilungen aus dem Materialprüfungsamt und dem Kaiser-Wilhelm-Institut für Metallforschung zu Berlin-Dahlem 41, 1923, S. 60-62.

46 Schiebold/Richter (wie Anm. 23), S. 91f.

47 E. Macherauch u. H. Neff, Allgemeine Entwicklung bis Mitte der 20erJahre, in: F. H. W. Heuck u. E. Macherauch (Hg.), Forschung mit Röntgenstrahlen: Bilanz eines Jahrhunderts (1895-1995), Berlin 1995, S. 325-351, hier S. 347 und G. Gottstein u. E. Nüsperling, Texturforschung mit Röntgenstrahlen, in: F.H.W. Heuck u. E. Macherauch (Hg.), Forschung mit Röntgenstrahlen: Bilanz eines Jahrhunderts (1895-1995), Berlin 1995, S. 462 472, hier S. 463.

48 K. Weißenberg, Mitteilungen aus dem Materialprüfungsamt und dem Kaiser-WilhelmInstitut für Metallforschung zu Berlin-Dahlem 1927, (Sonderheft III), S. 213-242.

49 M. Polanyi u. G. Sachs, Mitteilungen aus dem Materialprüfungsamt und dem KaiserWilhelm-Institut für Metallforschung zu Berlin-Dahlem 1926, (Sonderheft II), S. 188190 u. S. 191-200. 


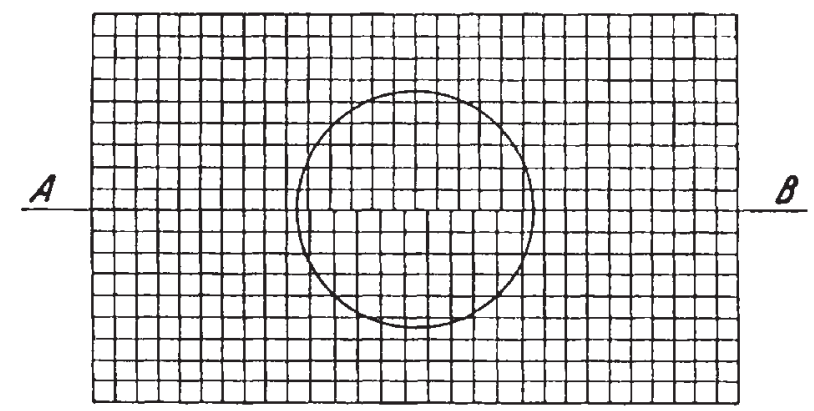

Abb. 6: Schematische Darstellung einer Gitterversetzung nach Polanyi. (Quelle: Zeitschrift für Physik 89, 1934, S. 661)

Metallforschungsinstitut zusammenarbeitete. Obwohl er sich nur relativ kurz mit dieser Thematik beschäftigt hatte (,My time with X-rays and crystals lasted three years ${ }^{\text {“50 }}$ ), deutete er im April 1932 das plastische Verhalten von Kristallen durch Stufenversetzungen, spezielle Kristallbaufehler. Abbildung 6 zeigt zweidimensional das Schema einer Gitterversetzung, das Polanyi erst nach zweijähriger Verzögerung publizierte. Dargestellt ist eine Störung des regelmäßigen Kristallaufbaus ,inmitten eines sonst unversehrten Gitters“. In dem kreisförmigen Ausschnitt sind entlang der Linie A-B ,die Atome gegeneinander versetzt“: zehn Atomen unterhalb der Linie „stehen elf Atome auf der anderen Seite ... gegenüber". ${ }^{51}$

Die Entdeckung der Stufenversetzung „bedeutete eine der wichtigsten Erkenntnisse in der Metallkunde, vielleicht sogar die wichtigste Erkenntnis auf diesem Gebiet überhaupt, " ${ }^{\text {52 }}$ die gleichwohl leider nicht mit einem Nobelpreis honoriert wurde..$^{53}$ Eine plastische Deformation bewegt sich danach im Kristall Stufe für Stufe auf atomarer Ebene zwischen dicht gepackten Schichten, indem Versetzungen zunächst erzeugt und dann verschoben werden. Die von Moellendorff 1913 postulierten ,größeren Bewegungen (vorwiegend Gleitungen) ${ }^{، 54}$ treten diskontinuierlich in atomaren Einzelschritten auf. Erst die Entdeckung der Stufenversetzungen erklärt strukturell das plastische Verhalten der Metalle, die Basis der uralten Technologien des Treibens, Hämmerns, Walzens und Schmiedens, und auch z.B. der Herstellung des für seine Schärfe, Zähigkeit und Härte berühmten Damaszener Stahls.

50 M. Polanyi, Fifty Years of X-Ray Diffraction, Oosthoek, Utrecht 1962, S. 629-636. http://www.iucr.ac.uk/iucr-top/publ/50YearsOfXrayDiffraction/polanyi.pdf.

51 M. Polanyi, Über eine Art Gitterstörung, die einen Kristall plastisch machen könnte, in: Zeitschrift für Physik 89, 1934, S. 660-662, hier S. 662.

52 W. J. Moore, Der feste Zustand, Eine Einführung in die Festkörperchemie anhand sieben ausgewählter Beispiele, Braunschweig 1977, S. 85.

53 H. Föll: http://www.techfak.uni-kiel.de/matwis/amat/def_en/kap_5/backbone/r5_1_1.html.

54 Moellendorff/Czochralski (wie Anm. 18), S. 1018. 
Der Ungar Polanyi, ähnlich Moellendorff von bemerkenswerter geistiger Vielseitigkeit, mit Engagement für Ökonomie und Politik, verließ 1933 wegen seiner jüdischen Abstammung Deutschland; dadurch verzögerte sich die Veröffentlichung seiner Entdeckung. Er trat in Manchester zunächst eine Professur für Physikalische Chemie, dann für Soziologie an. 1934 wurden zwei weitere unabhängige Untersuchungen über Stufenversetzungen zur Deutung der Kristallplastizität bekannt. ${ }^{55}$ Deshalb gelten heute Michael Polanyi, Egon Orowan und Geoffrey Taylor gleichermaßen als ihre Entdecker. Moellendorff wurde 1927 als Vertreter Deutschlands zum Vizepräsidenten des internationalen Verbands für Metallprüfung berufen ${ }^{56}$ und im gleichen Jahr zum Senator der KWG ernannt. 1929 trat er von der Amts- und der Institutsleitung zurück. 1933 wurde das KWI für Metallforschung aus finanziellen Gründen geschlossen und ein Jahr später mit neuer Finanzierung, neuen Forschungsaufgaben und wesentlich größerer Industrienähe in Stuttgart wieder gegründet.

\section{Rückzug und letzte Lebensjahre}

Handeln und Denken der Nationalsozialisten, die sich ab Januar 1933 Deutschlands bemächtigten, standen im völligen Widerspruch zu Moellendorffs Prinzipien. Für die Nazigrößen, „Kavaliere der abgebrochenen Lebensläufe", die meist an ehrlicher Berufsarbeit gescheitert seien, hatte er nur bitteren Spott. Der neue „politische Weg“ verlange an „Leistung nur Marschieren, Heilrufen und Schulterriemen zum Draufschlagen. ${ }^{657}$ Aufgrund des Gesetzes zur Wiederherstellung des Berufsbeamtentums vom April 1933 verloren viele Wissenschaftler jüdischer Abstammung ihre Stellen, eine erste Emigrationswelle lief durch Deutschland; Haber und Einstein waren unter den prominentesten Opfern der neuen Politik.

Enttäuscht über mangelnde Anerkennung seiner Arbeiten durch die Verwaltung der KWG schlug Moellendorff Anfang Mai 1933 dem Präsidenten der KWG, dem Physik-Nobelpreisträger Max Planck vor, seinen Senatssitz aufzugeben, wenn dies gewünscht sei. ${ }^{58}$ Allerdings wurde schon kurz darauf der Senat der KWG durch die neuen Machthaber genötigt, insgesamt zurückzutreten, „da eine Totalerneuerung gewünscht wurde. “59 Erneut schrieb Moellendorff darauf an Planck, ihm sei an einer Wiederberufung in den Senat in den

55 E. Orowan, Zur Kristallplastizität, in: Zeitschrift für Physik 89, 1934, S. 605-613, S. $614-$ 633 u. S. 634-659 und G. I. Taylor, The Mechanism of Plastic Deformation of Crystals, in: Proceedings of the Royal Society of London, 1934, S. 145, S. 362-387 u. S. 388-404. Braun (wie Anm. 27), S. 183.

57 Zitiert nach unveröffentlichten Aufzeichnungen von Dr. E. Kleinstück.

58 Briefkopien in BArch Koblenz, NL W. von Moellendorff, Nr. 1158/188.

59 H. Albrecht u. A. Hermann, Die Kaiser-Wilhelm-Gesellschaft im Dritten Reich (19331945), in: R. Vierhaus u. B. vom Brocke (Hg.), Forschung im Spannungsfeld von Politik und Gesellschaft: Geschichte und Struktur der Kaiser-Wilhelm-/Max-Planck-Gesellschaft, Stuttgart 1990, S. 356-406, hier S. 369f. 
herrschenden „bewegungsfreudigen Zeiten“ nicht gelegen. ${ }^{60}$ Zwei Monate später kündigte Moellendorff auch seine Mitgliedschaft in der KWG selbst: „Mitglied der Gesellschaft will ich nicht bleiben, da ich als ,Konservativer Sozialist ${ }^{`}$ zwar mancher heute modern gewordenen Idee verbunden, mit gewissen mir allmählich bekannt werdenden Vergewaltigungen aber, die weder mit der deutschen, noch mit der wissenschaftlichen Sache das Geringste zu tun haben, nicht einverstanden bin und nicht einmal scheinen mag." ${ }^{\text {"61 }}$

Damit gehört Moellendorff zu den wenigen, die sich nicht der Selbsttäuschung über den wahren Charakter des neuen Regimes hingaben, anders als viele andere der wissenschaftlichen und technischen Elite Deutschlands. Und er gehört zu denen, die dies auszusprechen wagten, obwohl er damit rechnen musste, dass seine Briefe über die Verwaltung der KWG auch an die Ministerien gelangten. Im Juli 1934 wurde das neue KWI für Metallforschung in Stuttgart eröffnet. Bei der Einweihungsfeier des Institutsneubaus 1935 wurde klar, wie die Metallforschung nun ausgerichtet werden sollte: Es waren ,neben dem Reichsstatthalter und prominenten Repräsentanten - allesamt namhafte Parteifunktionäre - auch ranghohe Militärs vertreten. “62 Max Planck, noch bis 1937 Präsident der KWG, wurde zugemutet, zur Einweihung an einem hakenkreuzgeschmückten Rednerpult zu sprechen. ${ }^{63}$ Der neue Direktor des Instituts, der Physikochemiker und Metallkundler Werner Kösters, gab im September 1936, schon vor der Verkündigung des Vierjahresplanes, an, wehrwichtige Forschung betrieben zu haben, z.B. die Einsparung von devisenbelasteten Metallen durch Verwendung heimischer Metalle. Auch würden neue Verfahren zur zerstörungsfreien Prüfung von Maschinengewehrläufen ausgearbeitet. $^{64}$ Von selbständiger Grundlagen-Metallforschung, wie Haber sie gefordert und Moellendorff und seine Mitarbeiter sie durchgeführt hatten, konnte jetzt keine Rede mehr sein. Nach dem Freitod seiner zweiten Ehefrau, der möglicherweise durch Verfolgungsmaßnahmen wegen ihrer jüdischen Abstammung ausgelöst war, setzte Moellendorff nur wenige Tage später im Mai 1937 im Alter von 56 Jahren seinem Leben selbst ein Ende.

60 Briefkopien in BArch Koblenz, NL W. von Moellendorff, Nr. 1158/188.

61 Ebd.

62 H. Maier, „Wehrhaftmachung“ und „Kriegswichtigkeit“, Zur rüstungstechnologischen Relevanz des Kaiser-Wilhelm-Instituts für Metallforschung in Stuttgart vor und nach 1945. Forschungsprogramm „Geschichte der Kaiser-Wilhelm-Gesellschaft im Nationalsozialismus" (Hrsg.: C. Sachse im Auftrag der Präsidentenkommission der Max-Planck-Gesellschaft zur Förderung der Wissenschaften e.V., Berlin), Ergebnisse 5, 2002, S. 13, http://www.mpiwg-berlin.de/KWG/Ergebnisse/Ergebnisse5.pdf

63 U. Kohl, Die Kaiser-Wilhelm-Gesellschaft zur Förderung der Wissenschaften im Nationalsozialismus, Quelleninventar, Veröffentlichungen aus dem Archiv zur Geschichte der Max-Planck-Gesellschaft, Bd. 10, Berlin, 1997.

64 Maier (wie Anm. 62). 


\section{Zusammenfassung}

Wichard von Moellendorff hat Technologie, Forschung und Entwicklung im AEG-Konzern von 1908 bis 1914 richtungsweisend beeinflusst und geprägt. Die Gliederung seines Metall-Laboratoriums in eine mechanische, eine chemische und eine metallographische Abteilung war über Jahrzehnte Vorbild für viele Laboratorien der Metallindustrie in Deutschland. Moellendorff löste die in den AEG-Betrieben „bis dahin souveräne Werkmeisterempirie“"65 durch Erkenntnisse aus systematischen Materialprüfungen ab und schlug die ersten „Legierungsnormalien“ vor.

Mit wachem Verstand, keckem Mut und großer Standfestigkeit hat er auf der Basis guter experimenteller Daten die wissenschaftliche Kontroverse mit einem der angesehensten Metallforscher seiner Zeit, Gustav Tammann, nicht gescheut und behielt Recht. Die aus heutiger Sicht größte Entdeckung, die im AEG-Metall-Laboratorium Oberspree gelang, das Einkristallziehen aus einem mit einer Schmelze gefüllten Tiegel (Czochralski-Verfahren), ist den durch Moellendorff gelegten methodischen Grundlagen mit zu verdanken.

Im Ersten Weltkrieg begann Moellendorff neben der Organisation der Kriegsrohstoffversorgung neue gesellschaftliche Perspektiven für die Zeit nach dem Kriege zu entwickeln. Mit seiner Vision einer Deutschen Gemeinwirtschaft scheiterte er an Parteien und Industrie und trat daraufhin als Unterstaatssekretär im Reichswirtschaftsministerium zurück. Doch wurde er 1926 in den Wirtschafts-Unterausschuss der vorbereitenden Abrüstungskonferenz in Genf berufen - und das als ehemaliger Referent im Reichskriegsministerium.

Von 1923 bis 1929 leitete Moellendorff in Personalunion das Staatliche Materialprüfungsamt und das Kaiser-Wilhelm-Institut für Metallforschung in Berlin und wurde daneben 1927 Vizepräsident des internationalen Verbands für Metallprüfung. Das junge Metallforschungsinstitut litt unter großen finanziellen Schwierigkeiten und wäre ohne die „Symbiose“ mit dem Materialprüfungsamt wahrscheinlich bald wieder geschlossen worden. Moellendorff hat es während dieser Zeit durch grundlegende Forschungen mit namhaften Persönlichkeiten zu einer der bedeutendsten Materialforschungsstätten der Welt gemacht. Röntgenbeugungsarbeiten des Instituts, damals die modernste und fundierteste Methode, trugen wichtige Teilergebnisse zur Entdeckung des Kristallbaufehlers der Stufenversetzung im April 1932 durch Polanyi bei. Diese Entdeckung krönte die Forschungsarbeiten, die Moellendorff 1913 mit metallographischen Untersuchungen an Zugproben begonnen und bei denen er erstmals ,größere Bewegungen (vorwiegend Gleitungen)“ der Metallatome, bei Erhaltung der Kristallstruktur, als Erklärung des plastischen Verhaltens von Metallen postuliert hatte.

$\mathrm{Zu}$ Unrecht in Vergessenheit geraten ist Wichard von Moellendorff nicht zuletzt als ein Senator der Kaiser-Wilhelm-Gesellschaft, der dem nationalso-

65 Briefkopien in BArch Koblenz, NL W. von Moellendorff, Nr. 1158/188. 
zialistischen Ungeist durch seinen Rückzug 1933 widerstand und dabei nicht bereit war, schweigend und heimlich abzutreten. Nicht alle Senatoren und Mitglieder der KWG, nicht alle Professoren und Wissenschaftler und nicht alle Industriellen zeigten in jener Zeit eine so aufrechte Haltung.

Anschriften der Verfasser: Prof. Dr. Jürgen Evers, Department für Chemie und Biochemie der Ludwig-Maximilians-Universität München, Butenandtstr. 5-13, D-81377 München, E-Mail: eve@cup.uni-muenchen.de; Dr. Ulrich von Möllendorff, Breite Str. 40, D-76135 Karlsruhe, E-Mail: uvm@planetinterkom.de; Dr. Ulrich Marsch, Gravelottestr. 2, D-81667 München, E-Mail: u.marsch@addcom.de. 
